Geneviève BOUCHER, «Espace littéraire et spatialisation de la littérature », @nalyses, automne 2007

\title{
Geneviève BOUCHER
}

\section{Espace littéraire et spatialisation de la littérature}

En 1955, Maurice Blanchot introduisait le concept d'espace littéraire, lieu symbolique condensant la positivité de la création et la négativité de la mort. Un demi-siècle plus tard, force est de constater que cette notion est utilisée par des chercheurs d'appartenance théorique diverse, dans des sens tout aussi variés. Une mise au point s'impose : qu'est-ce donc qu'un espace littéraire? Ce concept est-il opératoire? Peut-il nous aider à penser la particularité du littéraire, son mode de fonctionnement, son ouverture au dehors? C'est à ces questions que tente de répondre Qu'est-ce qu'un espace littéraire?, collectif dirigé par Xavier Garnier et Pierre Zoberman.

Si la notion d'espace littéraire se présente dans cet ouvrage comme une alternative à celle de champ littéraire, elle vise moins à se substituer au concept bourdieusien qu’à élargir la réflexion sociologique en accordant à la littérature un statut d'exception. Procédant de cette exception, l'espace littéraire serait ainsi, selon les responsables de l'ouvrage, la "pièce maîtresse pour une résistance à l'encerclement du littéraire par le "tout sociologique" » (p. 6). Cette nouvelle définition de l'espace littéraire a le mérite de proposer un entre-deux fertile entre la conception absolutiste de Blanchot, qui tend à isoler la littérature du monde ambiant, et la conception sociologique de Bourdieu, qui pèche par l'excès inverse en circonscrivant la littérature tout entière dans le social.

Les textes de ce collectif sont organisés autour de trois axes de réflexion, lesquels correspondent à trois manières de concevoir l'espace littéraire dans ses relations avec d'autres espaces. La première partie de l'ouvrage cherche à établir quels sont les rapports entre la littérature et les textes en général. Qu'est-ce qui fait d'un texte donné une œuvre littéraire? Quelle est la différence de nature entre l'espace textuel et l'espace littéraire? 
Geneviève BOUCHER, «Espace littéraire et spatialisation de la littérature », @nalyses, automne 2007

L'article de Xavier Garnier, qui ouvre l'ouvrage, est sans doute celui qui répond le plus rigoureusement à la question formulée dans le titre en s'attachant à définir la nature et les frontières de l'espace littéraire. Pour Garnier, l'espace littéraire se distingue de l'espace textuel en ce qu'il n'est pas donné d'emblée. Pour qu'apparaisse un espace littéraire, quelque chose doit advenir, un événement doit avoir lieu; le texte doit s'ouvrir au dehors et, selon une dynamique analogue à celle du dialogisme bakhtinien, enregistrer la rumeur ambiante. Autrement dit, l'espace textuel doit se laisser «féconder par la vie» (p. 24) afin de constituer un espace littéraire. Pierre Piret ajoute sa voix à la tentative de définition ouverte par Garnier. À travers l'analyse d'une partie du Soulier de satin de Claudel, il montre que l'espace littéraire s'institue en position d'exception — et donc, d'extériorité — par rapport au monde. Pour Piret, affirmer l'existence d'un espace littéraire, c'est postuler du même coup que la littérature possède un espace qui lui est propre et qui agit de manière distinctive. Ce caractère d'exception de la littérature est également la notion clé de l'article de Pascale Hellégouarc'h, qui aborde la question sous l'angle de l'intertextualité, procédé qui, par un jeu complexe de renvois et d'autoréférence, construit un espace littéraire dont les contours sont sans cesse mouvants. À partir d'une étude de la parodie et du pastiche, l'auteure montre que l'écriture mimétique pose la littérature comme un espace transversal constitué de croisements, un espace sans cesse construit et reconstruit selon la logique d'épaisseur temporelle qui est celle de l'intertextualité. Paul Allan Miller, pour sa part, aborde l'espace littéraire de manière contournée par une tentative de définition de la nature du littéraire. L'espace littéraire et la littérature sont ainsi, sous sa plume, une seule et même chose. Si l'on peut déplorer ce flou conceptuel, on retiendra tout de même de sa réflexion l'idée que si le littéraire et, par extension, l'espace littéraire, est construit par la «vie sociale », il ne représente pas moins l'atteinte d'un au-delà, la production d'un « surplus de sens ».

Après cette tentative de définition intrinsèque de l'espace littéraire, la deuxième partie de l'ouvrage explore les liens que l'espace littéraire entretient avec l'espace social. L'espace littéraire apparaît comme une sphère médiatrice à l'intérieur de laquelle les diverses entités du monde se livrent à un dialogue permanent. 
Geneviève BOUCHER, «Espace littéraire et spatialisation de la littérature », @nalyses, automne 2007

Pierre Zoberman interroge les relations que l'espace littéraire entretient avec l'espace social de même que la possibilité — ou l'impossibilité de concevoir un espace qui soit purement littéraire. En questionnant la notion de canon littéraire dans une perspective institutionnelle fortement inspirée de la sociologie bourdieusienne du champ, Zoberman montre que l'espace fonctionne comme une structure à l'intérieur de laquelle s'exercent des rapports de force qui président à l'inclusion ou à l'exclusion de certaines œuvres. Pour sa part, William J. Spurlin cherche moins à théoriser l'espace littéraire au sens large qu’à étudier le cas spécifique de l'Afrique du Sud après l'apartheid. Il met en lumière l'héritage paradoxal de la colonisation et de l'impérialisme en montrant que le traitement littéraire de l'orientation sexuelle a comme arrière-plan le clivage racial hérité de l'ère coloniale : le littéraire et le social se trouvent ainsi étroitement imbriqués. Enfin, Jean-Baptiste Voisin analyse le traitement de l'espace provençal dans l'œuvre de Giono. Plus précisément, il s'attache à cerner les enjeux éthiques impliqués dans le passage de l'utopie (imagination de liens entre l'espace réel et l'espace symbolique) à l'atopie (suspension du lien géographique au profit d'un symbolisme éthique). Cette notion d'atopie, où le lieu physique cède la place au lieu symbolique, serait selon l'auteur la plus à même de définir l'espace littéraire.

Enfin, dans la troisième partie, il est encore question des liens que l'espace littéraire entretient avec d'autres espaces, mais cette fois, c'est l'espace référentiel qui est mis en cause. La littérature est conçue comme une zone frontière, un espace de cohabitation, opérant une série de raccordements entre les différents espaces. Selon ce schéma, « la littérature serait l'opération par laquelle les espaces parviennent à entrer en contact les uns avec les autres sans chercher à s'absorber mutuellement » (p. 12).

Valérie Magdelaine-Andrianjafitrimo, en s'attachant au cas particulier de la littérature mauricienne, tente de différencier les notions de champ et d'espace littéraire. Si le champ littéraire opère un quadrillage à l'intérieur duquel agissent diverses forces, l'espace, lui, a une extension illimitée, permettant ainsi d'accueillir une parole littéraire irréductible au seul territoire national. Le concept d'espace littéraire 
Geneviève BOUCHER, «Espace littéraire et spatialisation de la littérature », @nalyses, automne 2007

aurait ainsi plus de souplesse que celui de champ, car il serait ouvert à l'échange des langues et des imaginaires de même qu'à l'interaction avec les espaces social, anthropologique et politique. Autre lieu de contacts interculturels, l'espace littéraire japonais tel que décrit par Marc Kober subit la «contamination» d'autres espaces et d'autres identités. Selon Kober, dans le contexte actuel de mondialisation, les romans japonais traduits en français reflètent non seulement la diminution de l'appartenance culturelle au profit d'une neutralité spatiale plus facilement exportable, mais également la diminution de l'appartenance à la littérature "pure » par la greffe, voire l'envahissement d'autres espaces (cinématographique, musical, médiatique, technologique). Enfin, Maarten van Delden ferme la marche en abordant l'espace littéraire sous l'angle de la délimitation territoriale. Il se penche sur deux récits mexicains qui problématisent la question de la frontière entre les États-Unis et le Mexique. Si la notion de confins permet de dépasser le clivage géopolitique traditionnel et d'envisager un espace de mélange et de chevauchement, les deux récits étudiés ne montrent pas moins que la proximité de la frontière renforce plus qu'elle n'atténue la conscience d'une différence culturelle.

Le principal reproche que l'on peut faire à cet ouvrage est sans doute son manque d'homogénéité, surtout en ce qui a trait à la notion thème. L'érudition déployée et la finesse générale des analyses ne parviennent pas à faire oublier ce point essentiel : au bout de ces 206 pages, on ne sait pas plus ce qu'est un espace littéraire. Voilà un détail gênant pour un ouvrage dont le titre nous fait espérer une réponse, ne serait-ce que partielle. Si les responsables de l'ouvrage dénoncent en introduction le flou conceptuel entourant la notion d'espace littéraire, force est de constater qu'ils tombent eux-mêmes dans le piège de cette polysémie. Tout se passe comme si, dans la pratique, le concept d'espace littéraire était d'autant plus opératoire qu'il était défini de manière imprécise.

Mais le plus dérangeant n'est pas cette multiplicité de définitions : c'est le flou épistémologique entourant la compréhension même de l'espace littéraire et de son champ d'action. Trois tendances diamétralement opposées se côtoient au sein du collectif. La première tendance est, à nos yeux, la plus pertinente et, surtout, la plus rigoureuse sur le plan 
Geneviève BOUCHER, «Espace littéraire et spatialisation de la littérature », @nalyses, automne 2007

conceptuel. Elle part du principe que l'espace littéraire n'est assimilable ni à l'espace référentiel ni à l'espace textuel. Sans s'y limiter, elle invite à réfléchir sur la nature du littéraire afin de mieux comprendre quel est le «lieu» de la littérature, quel est cet «espace surnuméraire » (p. 8) ouvert par les œuvres.

Une deuxième tendance épistémologique s'inscrit dans la lignée des études postcoloniales. Les auteurs qui conçoivent l'espace littéraire de cette manière s'intéressent à la production littéraire d'un pays ou d'un territoire donné; leur objet d'étude porte sur les relations (d'adhésion ou d'opposition) que cette production entretient avec la culture hégémonique, qu'elle soit celle de l'ancien colonisateur, du nouveau pouvoir en place ou, plus globalement, d'une normalisation issue de la mondialisation. Le point faible de cette tendance est de confondre le concept d'espace littéraire avec l'espace géographique. Si, comme il est énoncé en quatrième de couverture, la notion d'espace littéraire " ouvre des perspectives nouvelles aux études postcoloniales », elle ne s'y limite pas. Ce qui pose problème dans cette approche est que la notion d'espace littéraire se trouve assimilée à celle d'une littérature nationale plus ou moins affirmée. L'espace littéraire comme concept se dissout dans l'univers référentiel jusqu'à désigner la détermination spatiale d'une littérature donnée. Cette confusion est peut-être attribuable au fait que la plupart des auteurs du collectif sont affiliés au CENEL (Centre d'étude des nouveaux espaces littéraires) de l'Université Paris 13, qui était jusqu'en 2005 le Centre d'études francophones et comparées. Le fait de transformer un centre de recherche sur les littératures francophones et comparées en un centre d'étude des nouveaux espaces littéraires ne contribue-t-il pas à assimiler espace littéraire et détermination géographique? Ces «nouveaux espaces littéraires» qui constituent l'objet de recherche du centre peuvent-ils être assimilés à l'espace littéraire, au singulier, tel qu'il apparait dans le titre de l'ouvrage?

À côté de cette tendance à la territorialisation se trouve une troisième voie qui consiste à amalgamer naïvement l'espace littéraire à la représentation de l'espace dans les œuvres. Elle s'exprime le plus fortement dans des textes comme celui de Maarten Van Delden, qui 
Geneviève BOUCHER, «Espace littéraire et spatialisation de la littérature », @nalyses, automne 2007

traite de la manière dont l'espace frontalier entre les États-Unis et le Mexique est représenté dans les œuvres. Ce n'est plus d'espace littéraire qu'il s'agit alors, mais d'espace dans la littérature. En introduction, Xavier Garnier et Pierre Zoberman font remarquer que, selon un curieux phénomène de mise en abyme, l'espace littéraire est souvent traité par le biais de textes qui parlent de l'espace physique. Mais s'agit-il vraiment d'une mise en abyme? Ne doit-on pas plutôt y voir une confusion entre deux types de spatialisation, l'un conceptuel et l'autre thématique?

Si l'idée de définir un espace littéraire spécifique, conçu à la fois comme «lieu» de la littérature et comme carrefour entre différents espaces, est tout à fait pertinente, force est de constater que le caractère malléable de la notion fait courir le risque d'associer tout et n'importe quoi à l'espace littéraire et de mettre dans un même panier l'espace référentiel ou géographique, le lieu de production des œuvres et la thématisation de l'espace dans les œuvres. Ce danger, l'ouvrage dirigé par Garnier et Zoberman ne semble pas l'avoir évité. Bien que quelques-uns des auteurs de ce collectif offrent une précieuse contribution en vue d'une future théorisation de l'espace littéraire, l'ensemble déçoit par son hétérogénéité et son manque de rigueur conceptuelle. Voilà donc un ouvrage qui laisse ouverte - c'est un euphémisme - la question à laquelle il s'était promis de répondre, mais qui a tout de même le mérite de proposer une ouverture, un renouvellement de la notion d'espace littéraire, notion qui, à l'origine, était très peu applicable aux approches sociologiques de la littérature. Si l'ouvrage n'offre que peu de réponses satisfaisantes, il fait, par son échec même, la démonstration paradoxale du flou théorique entourant le concept et, par conséquent, du bien-fondé de la question initiale.

Référence : Xavier Garnier et Pierre Zoberman, Qu'est-ce qu'un espace littéraire?, Saint-Denis, Presses universitaires de Vincennes, coll. «L'imaginaire du texte », 2006, 206 p. 